\title{
Europapolitik - kein vorrangiges Thema im französischen Präsidentschaftswahlkampf
}

\author{
Franziska Brantner und Anja Thomas*
}

Die französischen Präsidentschaftswahlen werden seit dem Nein zum Vertrag über eine Verfassung für Europa (VVE) in Frankreich und den Niederlanden im Mai 2005 mit Spannung erwartet. Eine Kursbestimmung für die Zukunft Europas wird nach Meinung vieler Experten erst wieder mit einem neuen französischen Präsidenten angegangen werden können.

Vor diesem Hintergrund stellt sich die Frage, welchen Stellenwert ,Europa' gegenwärtig in der öffentlichen Debatte Frankreichs hat, welche Vorschläge für die Zukunft des Verfassungsvertrages diskutiert und welche europapolitischen Themen in den beiden großen und gemäßigten Lagern im französischen Präsidentschaftswahlkampf eine Rolle spielen werden. Welche Positionen beziehen die Präsidentschaftskandidaten Sarkozy und Royal? Wird der zu erwartende politische ,Generationenwechsel' in Paris zu deutlichen Veränderungen in der französischen Europapolitik führen oder wird Kontinuität überwiegen?

\section{Leitbilder und Konfliktlinien der französischen Europapolitik}

Grundsätzlich lassen sich zwei Entwicklungen ausmachen: Zum einen kristallisiert sich ein Wendepunkt in der französischen Europadebatte heraus. Seit dem Referendum von Maastricht hat sich die entscheidende europapolitische Konfliktlinie gewandelt. ${ }^{1}$,Europa im Sinne der EU wird in der französischen öffentlichen Debatte als politischer Handlungsrahmen akzeptiert. Die Debatte verlagert sich auf Kontroversen darüber, wie diese Europäische Union aussehen soll. Dabei steht das Bild eines vermeintlich ,neoliberalen " der Forderung nach einem ,sozialen' Europa in der französischen öffentlichen Diskussion diametral gegenüber. Den an Einfluss verlierenden ,Souveränisten' steht eine neue Gruppe von ,Europaskeptikern' gegenüber, die eine supranationale Integration der Europäischen Union zwar befürwortet, die heutige Europäische Union jedoch für ein demokratisch defizitäres, neoliberales Gebilde hält.

Neben dieser durchaus bedeutenden Verschiebung lassen sich jedoch auch während des aktuellen Präsidentschaftswahlkampfes Kontinuitäten in der Debatte erkennen, die an traditionelle französische Europadebatten anknüpfen. Im Mittelpunkt der Diskussion stehen die Wirtschafts- und Sozialpolitik und die Rolle Europas in der Welt. Die Erweiterung und die damit verbundene Sorge, in der EU sozialpolitisch bedroht und machtpolitisch marginalisiert zu werden, ist ein Querschnittsthema, dass in diese Einzeldebatten in Frankreich hineinspielt.

In der Debatte dominieren weiterhin Leitbilder der ,Europe puissance“ und des ,Europe sociale', die dem traditionellen französischen Leitbild von der Rolle Europas in der Welt als Gegengewicht zu den USA entsprechen und versuchen, das französische Sozialstaatsver-

\footnotetext{
Franziska Brantner, M.A., wissenschaftliche Mitarbeiterin, European Studies Centre, University of Oxford. Anja Thomas, M.A., wissenschaftliche Mitarbeiterin, Jean Monnet Lehrstuhl, Forschungsinstitut für Politische Wissenschaft und Europäische Fragen, Universität zu Köln.
}

1 Vgl. Joachim Schild: Ein Sieg der Angst - das gescheiterte französische Verfassungsreferendum, in: integration 3/2005, S. 187-200, hier S. 192. 
ständnis auf die Europäische Union zu übertragen. Forderungen nach einem Europa, das vor der Globalisierung ,schützt ' statt sie zu verstärken haben sich im Zuge des gescheiterten Verfassungsreferendums in allen Lagern weit verbreitet. Dies zeigen besonders Äußerungen von Nicolas Sarkozy, der seine ursprünglich ,liberaleren' und kritischeren Positionen zum bestehenden französischen Wirtschafts- und Gesellschaftsmodell stärker auf den Diskurs über ein soziales Europa ausrichtet und somit abschwächt. ${ }^{2}$ Das Repertoire von auf europäischer Ebene schwer umsetzbaren Forderungen ist dementsprechend lang.

Hier zeigt sich eine zweite Kontinuität: Die EU wird in Frankreich nur dann als Erfolg gepriesen, wenn sie der, vision francaise' Europas entspricht. Einer der wichtigsten Punkte der Referendumsdebatte war die Frage, inwieweit der Verfassungsvertrag der französischen Vision der ,Europe puissance ‘ und des ,Europe sociale“ entspricht - entsprechende Kompromisse wurden selbst von Verfassungsbefürwortern allenfalls als , hinzunehmendes Übel ‘ beschrieben. ${ }^{3}$ Statt Kompromisse zu akzeptieren und als unvermeidbaren Prozess in einer Union von (damals) 25 Staaten zu rechtfertigen, versprachen Verfassungsbefürworter, in Zukunft sicherzustellen, dass die EU mehr den Vorstellungen Frankreichs entsprechen werde. Eine ähnliche Rhetorik wiederholt sich nun auch im Wahlkampf.

\section{Der Präsidentschaftswahlkampf in Frankreich}

\section{Die Kandidaten}

Die Präsidentschaftskandidaten der beiden großen politischen Lager ${ }^{4}$, Nicolas Sarkozy, gegenwärtig Innenminister der konservativen Regierungspartei Union pour un Mouvement Populaire (UMP) und die Kandidatin der Parti Socialiste (PS), Ségolène Royal, die vor einem Jahr noch als Außenseiterin galt, sich aber gegen Laurent Fabius und Dominique Strauss-Kahn durchsetzte, sollen hier vorgestellt werden.

Entscheidend beeinflussen könnte den Wahlausgang, ob Jean-Marie Le Pen, Kandidat der rechtsextremen Front National, die erforderlichen Unterschriften zur Unterstützung seiner Kandidatur von gewählten Repräsentanten erhalten wird. Nachdem Le Pen 2002 in die zweite Runde der Präsidentschaftswahl eingezogen war wird vermutet, dass potenzielle Unterstützer diesmal zögerlicher sein werden. Vor der letzten Wahl hatte er nur 33 mehr als die 500 erforderlichen Patenschaften für seine Kandidatur erhalten. ${ }^{5}$ Die Erinnerung an die Präsidentschaftswahl 2002 könnte bereits im ersten Wahlgang zu einer Konzentration der Stimmen auf die beiden Favoriten führen. ${ }^{6}$

2 Vgl. Institut für Europäische Politik (Hrsg.): EU-25 Watch No. 2, Berlin 2006, hier S. 235.

3 Vgl. Nicolas Jabko: La France face à la Constitution européenne: un héritage mal assumé, in: Critique internationale 29/2005, S. 135-149, hier S. 137-138.

4 Die traditionell pro-europäische Union pour la démocratie française (UDF) tritt mit ihrem Kandidaten François Bayrou an. Die Grünen haben sich auf die ehemalige Umweltministerin unter Lionel Jospin, Dominique Voynet, als Kandidatin geeinigt. Sie bekommen im Bereich der Umweltpolitik jedoch Konkurrenz von Nicolas Hulot, einem parteiunabhängigen Umweltrechtler, der mit einer möglichen Kandidatur spielt und in Umfragen überdurchschnittlich gut abschneidet. Links der Sozialisten wird eine gemeinsame Kandidatur angestrebt, die sich hauptsächlich aus Gegnern des Verfassungsvertrages zusammensetzt. Die kommunistische Partei hat bereits ihre Parteivorsitzende Marie-George Buffet zur Kandidatin gekürt. Es sind jedoch weitere parallele Kandidaturen zu erwarten. Im Lager der extremen Rechten haben sich Philippe de Villiers (Mouvement pour la France) und Jean-Marie Le Pen (Front national) bereits offiziell zu Kandidaten erklärt.

5 Vgl. Le Monde: Le Conseil constitutionnel ne publiera plus la liste complète des parrainages, 02.11.2006.

6 LeMonde.fr : Les intentions de vote au premier tour, Ipsos-Umfrage ,Le Point“ vom 01./02.12.2006, 07.12.06, abrufbar unter: http://lemonde.fr/web/infog/0,47-0@2-823448,54-842864,0.html (letzter Zugriff: 13.12.2006). 


\section{Europa als Thema im Wahlkampf}

Außenpolitik und im Speziellen die Europapolitik sind für die großen Parteien in Frankreich nicht identitätsstiftend. ${ }^{7}$ Sie spielen deshalb in den französischen Präsidentschaftswahlkämpfen traditionell eine untergeordnete Rolle, obwohl dem Präsidenten laut Verfassung eine starke Position in der Außen- und Sicherheitspolitik und damit auch in der Europapolitik zukommt, die nur in Zeiten der Kohabitation abgeschwächt wird. Er entscheidet im Wesentlichen über die europapolitischen Positionen Frankreichs. ${ }^{8}$

Die europäische Integration ist auch kein vorrangiges Thema des bisherigen Wahlkampfes. So kommt in der Antrittsrede Royals als Kandidatin der PS der Begriff ,Europäische Union" nicht vor. ${ }^{9}$ Die konservative UMP versucht allerdings, dieses Thema zu lancieren und hofft, dass es im Nachgang des gescheiterten Verfassungsreferendums politischen Sprengstoff für das sozialistische Lager entfalten wird. Sarkozy zwang die Sozialisten durch zwei Grundsatzreden zur Zukunft Europas ${ }^{10}$ zu einer ersten Positionierung. Royal präsentierte daraufhin ihre Vorstellungen in einem ,Sieben-Punkte-Plan` vor Journalisten im Rahmen einer Plenarsitzung der Assemblée nationale. ${ }^{11}$

\section{Vorstellungen für die Zukunft der Europäischen Union}

Nach dem gescheiterten Referendum zum Vertrag über eine Verfassung für Europa im Mai 2005 ist die Frage nach dem weiteren Voranschreiten der europäischen Integration oder nach möglichen Wegen aus der Krise das Kernthema, auf das die europapolitische Debatte im Wahlkampf in Frankreich immer wieder zurückkommt.

Nach außen hatten französische Politiker schnell signalisiert, dass es mit Frankreich keine ,einfachen “ Wege aus der ,Verfassungsfalle“12 geben würde. ${ }^{13}$ Eine erneute Abstimmung über denselben Text wurde vom französischen Staatspräsidenten Jacques Chirac von Anfang an abgelehnt. Er betonte, dass die Situation in Frankreich insofern nicht mit dem irischen Präzedenzfall anlässlich der Ratifikation des Vertrags von Nizza vergleichbar sei. ${ }^{14}$ Diese Meinung wird immer noch lagerübergreifend geteilt. Nur der Präsident des Konvents und ehemalige Präsident Frankreichs, Valery Giscard d'Estaing, hält noch öffentlich am Verfassungsvertrag in seiner jetzigen Form fest. ${ }^{15}$ In der Bevölkerung ist die Ablehnung des

7 Vgl. Sylvie Goulard: Französische Europapolitik und öffentliche Debatte in Frankreich, Zentrum für Europäische Integrationsforschung, Paper C62/2000, S. 4.

8 Vgl. Gisela Müller-Brandeck-Bocquet: Frankreichs Europapolitik, Wiesbaden 2004, S. 16-23.

9 Vgl. Discours de Ségolène Royal au Congrès d'investiture du Parti Socialiste à la Mutualité, 26.11.2006, abrufbar unter: http://www.desirsdavenir.org/index.php?c=interventions\&id=461 (letzter Zugriff: 28.12.2006).

10 Vgl. Rede von Nicolas Sarkozy vor der Konrad-Adenauer-Stiftung und der Deutschen Gesellschaft für Auswärtige Politik, 16.02.2006, abrufbar unter: www.botschaft-frankreich.de, Frankreich-Info (letzter Zugriff: 17.03.2006); Rede von Nicolas Sarkozy vor den Amis de l'Europe und der Fondation Schuman, Brüssel, 08.09.2006, abrufbar unter: http://www.robert-schuman.org/actualite/bruxelles/discours8sept.pdf (letzter Zugriff: 25.11. 2006).

11 Vgl. Pressekonferenz von Ségolène Royal: „L'Europe par la preuve (avec les 7 propositions)“, Assemblée Nationale, 11.10.2006, abrufbar unter: http://www.desirsdavenir.org/index.php?c=interventions\&id=191 (letzter Zugriff: 25.11. 2006).

12 Udo Diedrichs/Wolfgang Wessels: Die Europäische Union in der Verfassungsfalle? Analysen, Entwicklungen und Optionen, in: integration 4/2005, S. 287-306, hier S. 287.

13 Vgl. Schild: Ein Sieg der Angst, S. 187.

14 Vgl. Pressekonferenz von Jacques Chirac am Ende des Europäischen Rates, Brüssel, 17.06.2005.

15 Vgl. Valéry Giscard d'Estaing: La Constitution européenne n'est pas morte, Interview mit der Redaktion von Toute 1'Europe, 26.10.2006, abrufbar unter: http://www.touteleurope.fr/fr/actualite-europeenne/questions-a/ valery-giscard-destaing.html (letzter Zugriff: 10.11.2006). 
aktuellen Textes weiterhin hoch. 74 Prozent der Gesamtbevölkerung lehnen selbst eine teilweise Umsetzung des Verfassungsvertrages ohne neues Referendum ab. ${ }^{16}$

Die Debatte, die dem Verfassungsreferendum voranging, markierte dennoch einen entscheidenden Wendepunkt der öffentlichen Debatte in Frankreich und der sie dominierenden Leitbilder um die Europäische Union. Es zeigte sich, dass seit 1992 eine Veränderung der entscheidenden Konfliktlinien der Europapolitik stattgefunden hatte. Beim Referendum zum Vertrag von Maastricht dominierte in der öffentlichen Diskussion die Konfliktlinie zwischen den überwiegend dem gaullistischen Lager und der nationalen Linken angehörenden ,Souveränisten ' und dem europabefürwortenden überwiegend mitte-links angesiedelten Lager unter Führung von François Mitterrand. ${ }^{17}$ Während erstere in der ,Nation ' den entscheidenden politischen Handlungsrahmen sahen und vor einer Übertragung von weiteren Kompetenzen auf die europäische Ebene warnten, sahen die Europabefürworter in der europäischen Integration die Möglichkeit, nach der deutschen Wiedervereinigung französische Interessen auf der europäischen und internationalen Ebene zu wahren. ${ }^{18}$

Die souveränistische Argumentationsweise findet sich aktuell vor allem noch bei den Parteien des extremen rechten und linken Flügels ${ }^{19}$ und vereinzelt in der UMP. In der Debatte um den Verfassungsvertrag zeigte sich jedoch, dass ein großer Teil der Verfassungsgegner nicht aus integrationsskeptischen Motiven handelte, sondern lediglich eine ,andere Europäische Union forderte. Im Diskurs der Verfassungsgegner stand besonders ein gewünschtes ,soziales“ Europa dem vermeintlich im Verfassungsvertrag festgeschriebenen ,neoliberalen' Europa gegenüber. Prominente Verfassungsgegner wie Laurent Fabius bei den Sozialisten forderten im Gegenteil sogar, die Europäische Union solle mehr supranationale Kompetenzen erhalten, um eine europäische Sozialpolitik besser und kohärenter vorantreiben zu können. ${ }^{20}$ Wenn diese neue Konstellation der politischen Fronten sicherlich auch der Tatsache geschuldet war, dass ein neo-gaullistischer Präsident und nicht wie 1992 ein sozialistischer Präsident den Verfassungsvertrag zur Abstimmung stellte, so schien und scheint sich doch abzuzeichnen, dass die EU als notwendiger Handlungsrahmen französischer Politik im öffentlichen Diskurs weitgehend akzeptiert wird. Dies zeigt sich auch in entsprechenden Umfragen in der Bevölkerung. Trotz des gescheiterten Referendums sprechen sich 67 Prozent der Franzosen generell für die Notwendigkeit einer Verfassung für die Europäische Union aus. ${ }^{21}$

Im Präsidentschaftswahlkampf schlagen die beiden Favoriten als Ausweg aus der konstitutionell-institutionellen Malaise der Europäischen Union zunächst jedoch vor allem pragmatische kurzfristige Lösungen vor. ${ }^{22}$ Sarkozy präsentierte im Februar 2006 in Berlin einen

16 Die von „Open Europe“ durchgeführte Umfrage ergab, dass 74\% der Interviewten meinen, selbst eine teilweise Umsetzung des VVE ohne neues Referendum dürfte den Politikern nicht erlaubt sein, abrufbar unter: www.openeurope.org.uk/mediacentre/pressrelease.aspx?pressreleaseeid=18 (letzter Zugriff: 30.05.2006). Die Ergebnisse der jüngsten Eurobarometer-Umfrage (Dezember 2006) deuten allerdings darauf hin, dass die Zustimmung in der französischen Öffentlichkeit gestiegen ist. 56\% der Befragten sind laut Eurobarometer für den Verfassungsvertrag. Abrufbar unter: http://ec.europa.eu/public_opinion/archives/eb/eb66/eb66_highlights_de.pdf (letzter Zugriff: 09.01.2007).

17 Vgl. Müller-Brandeck-Bocquet: Frankreichs Europapolitik, S. 118-122.

18 Ebenda, S. 88-96.

19 Vgl. Franziska Brantner/Michael Knoll: Constitution ,,attac-ed“: Nach dem gescheiterten Referendum zur EUVerfassung in Frankreich, in: Kommune - Forum für Politik, Ökonomie und Kultur 4/2005, S. 29-32.

20 Vgl. Schild: Ein Sieg der Angst, S. 193.

21 Vgl. La Croix: Comment relancer l'Europe, 29.05.2006, abrufbar unter : http://www.la-croix.com/documents/doc.jsp?docId=2270452\&rubId=786 (letzter Zugriff: 31.05 .2006 )

22 Vgl. beispielsweise Le Figaro: Tribune de M. Jacques Chirac, Président de la République, 26.10.2005, abrufbar unter: http://www.elysee.fr/elysee/francais/interventions/interviews_articles_de_presse_et_interventions_ televisees./2005/octobre/tribune_de_m_jacques_chirac_president_de_la_republique_parue_dans_le_figaro_et _plusieurs_journaux_europeens.31690.html (letzter Zugriff: 21.10.2006). 
Dreistufenplan, den er im folgenden September weiter ausführte. In einem ersten Schritt sollten diejenigen institutionellen und prozeduralen Verbesserungen umgesetzt werden, die nicht einer Ratifizierung bedürften. Anschließend solle ein kurzer institutioneller Vertrag, der den bestehenden Vertrag von Nizza modifiziere, eine Reihe institutioneller Veränderungen des VVE übernehmen, wie die Ausweitung der qualifizierten Mehrheit, die Einführung der , doppelten Mehrheit‘ im Ministerrat, die Wahl des Kommissionspräsidenten durch das Parlament, die Einführung eines Präsidenten des Europäischen Rates sowie die Stärkung nationaler Parlamente.

Dieser „traité pour un meilleur fonctionnement de l'Union“23 solle während der deutschen Ratspräsidentschaft verabschiedet und dann während der französischen Ratspräsidentschaft von den nationalen Parlamenten ratifiziert werden. In einem letzten Schritt solle dann eine breite und demokratische Debatte über die Ziele, Grenzen und Aufgaben der EU stattfinden, die in einen neuen Verfassungskonvent einmünden könnte. Für diese Phase bringt Nicolas Sarkozy neue Vorschläge ein, so zum Beispiel die Einführung einer ,Supermehrheit` (70-80 Prozent der gewogenen Stimmen im Ministerrat), die das Einstimmigkeitsverfahren in einzelnen politisch hochsensiblen Bereichen ersetzen würde, und die Bestimmung der Kommissionsmitglieder durch den Kommissionspräsidenten.

Royal unterstreicht die hohe Bedeutung einer erneuten Orientierungsdebatte zu Zielen der EU und die Entwicklung von konsensfähigen Politiken, die den Bürgern das europäische Projekt wieder näher bringen können („L'Europe par la preuve“). ${ }^{24}$ Diese ,funktionalistische" Herangehensweise birgt weniger politischen Sprengstoff für die eigene Partei. ${ }^{25}$ Die Kandidatin ist dem Parteimanifest der PS verpflichtet. Das Wahlprogramm zur Präsidentschaftswahl und zur Wahl der Assemblée nationale war Anfang Juli 2006 in der während der Referendumskampagne gespaltenen Partei überraschend im Konsens verabschiedet worden. ${ }^{26}$ Die Sozialisten lehnen darin die ,Wiedervorlage“ des Vertrags über eine Verfassung für Europa ab, selbst wenn dieser mit einer neuen Präambel ausgestattet sein sollte. Auch die Umsetzung einzelner Teile des VVE wird abgelehnt. Nach der Devise ,le traité constitutionnel est mort - vive la constitution!“27 schlagen sie stattdessen die Ausarbeitung eines neuen Verfassungsvertrages vor, der sich auf die institutionelle Organisation der EU konzentriert. Dabei heben sie besonders die Stärkung des Europäischen Parlamentes hervor. ${ }^{28}$ Dies würde denjenigen aus der ,Nein'-Kampagne in Frankreich entgegenkommen, die vor allem in den konkreten Regeln für einzelne Politikbereiche des Teil III des VVE unter der Etikette, Verfassung ' eine Gefahr für die demokratische (und soziale) Funktionsweise der Europäischen Union sahen. ${ }^{29}$

Bei einem Treffen mit dem luxemburgischen Premierminister Jean-Claude Juncker im Januar 2007 deutete Royal allerdings erstmals an, dass es mit ihr 2009 ein erneutes Referendum über einen ,traité fondmanetal de base' geben könne, wenn sozialpolitische und arbeits-

23 Rede von Nicolas Sarkozy vor den Amis de l'Europe, S. 2.

24 Vgl. Pressekonferenz von Ségolène Royal: L'Europe par la preuve (avec les 7 propositions).

25 Vgl. zu verschiedenen Herangehensweisen an die Verfassungskrise Anne Faber/Wolfgang Wessels: Strategien und institutionelle Perspektiven nach der Verfassungskrise: „Funktionalistische“ und ,,institutionalistische“ Wege zu einem neuen europäischen Verhandlungspaket, in: Politische Vierteljahresschrift 2/2006, S. 252-263, hier S. 258.

26 Vgl. Antoine Colombani: Modernisation or Reconciliation? The New Agenda of the French Socialists, 2006, abrufbar unter: http://www.policy-network.net/php/article.php?sid=3\&aid=639 (letzter Zugriff: 20.11.2006).

27 Vgl. Diedrichs/Wessels: Die Europäische Union in der Verfassungsfalle?, S. 293.

28 Vgl. Parti Socialiste: Réussir ensemble le changement. Le projet socialiste pour la France, abrufbar unter: http://projet.parti-socialiste.fr (letzter Zugriff: 07.11.2006).

29 Vgl. l'Humanité: Le discours sur l'impossibilité de la renégociation relève du mensonge, 11.05.2006. 
rechtliche Aspekte unterstrichen würden. Trotz der Unterschiede zwischen den Vorschlägen von Royal und Sarkozy zeigt sich eine entscheidende Übereinstimmung. Beide Kandidaten für das Präsidentenamt fordern auch nach dem gescheiterten Verfassungsreferendum als notwendige institutionelle Reformen nicht nur die im Konvent von Frankreich geforderte Einführung des Präsidenten des Europäischen Rates, sondern vor allem auch die Stärkung des Europäischen Parlaments. Sarkozy fordert darüber hinaus die Einführung der, doppelten Mehrheit‘. In beiden Punkten war Jacques Chirac bereits im Konvent zu Zugeständnissen bereit gewesen, die von den traditionellen Positionen Frankreichs abwichen. ${ }^{30}$ In der Tradition de Gaulles stand Frankreich im europäischen Integrationsprozess für das Prinzip eines intergouvernementalen Europas der Nationen ein und lehnte die Stärkung supranationaler Elemente der institutionellen Architektur ebenso ab wie die Ausweitung der qualifizierten Mehrheit. ${ }^{31}$ Zur Erreichung übergeordneter politischer Ziele - wie dem Schulterschluss mit Deutschland in der Irak-Krise und weiterer Schritte in der Europäischen Sicherheits- und Verteidigungspolitik in Richtung auf das von Frankreich seit langem verfolgte Leitbild der ,Europe puissance“ als Gegengewicht zu den Vereinigten Staaten - war Chirac im Konvent jedoch zu größeren Zugeständnissen vor allem an Deutschland bereit gewesen. ${ }^{32}$

Bei der Abweichung von traditionellen französischen Positionen könnte jedoch im aktuellen Präsidentschaftswahlkampf noch ein anderes Motiv eine Rolle spielen: Umfragen nach dem gescheiterten Referendum zeigen, dass die französische Bevölkerung eine Stärkung des Europäischen Parlaments mehrheitlich befürwortet. ${ }^{33}$ Dass Frankreich nun aber noch nicht „,vollständig in das Lager der Supranationalisten übergelaufen sei““34, zeigt sich dann, wenn man die Vorschläge der Parteien für die langfristige Lösung der Verfassungsfrage genauer betrachtet.

Das UMP-Wahlprogramm für 2007, auf das Sarkozy als Parteivorsitzender einen erheblichen Einfluss hatte, spricht im Gegensatz zu seiner Brüsseler Rede nicht mehr von einem neuen Verfassungsprozess, sondern nur von einem Reflexionsprozess, der in der Erarbeitung eines Textes münden soll, der die ,fundamental politische Dimension des europäischen Projektes besiegelt““.35 Dies weist eher auf das Aufgeben der Idee eines ,Verfassungstextes“ durch die französischen Konservativen hin. Beobachter vermuten, dass Sarkozy die Verfassungsidee in seiner Brüsseler Rede aufnahm, um sein „Image des Euroskeptikers [zu] zerschmetter[n], das ihm in gewissen Kreisen hartnäckig anhaftete “. ${ }^{36}$ Außerdem könnte er darauf hoffen, dass ihm während seiner Amtszeit ein entsprechendes Referendum erspart bliebe.

Die Debatte um langfristige Lösungen innerhalb des linken Lagers zeichnet sich oftmals durch eine Kluft zwischen Forderungen und europapolitischer Realität aus. Ob ein kurzer institutioneller Verfassungsvertrag, wie vom Parteiprogramm gefordert, auf der europäischen Ebene ausreichende Unterstützung finden könnte, ist fraglich. Gleichzeitig scheinen die ,neuen Euroskeptiker` als , bekennende Europäer' Vergemeinschaftungen dort zu fordern,

30 Müller-Brandeck-Bocquet: Frankreichs Europapolitik, S. 266.

31 Vgl. Hans Stark: Frankreichs Rolle in der Welt, in: Frankreich - Informationen zur politischen Bildung, Heft 285, 2004.

32 Müller-Brandeck-Bocquet, Frankreichs Europapolitik, S. 266.

33 Gesa-Stefanie Brincker: Country study: Leitbilder for the enlarged European Union in France, Studie im Rahmen des Forschungsprojekts „Leitbilder (key concepts) for the European Union after Enlargement: Fragmentation, Continuity or Reinvention?", Institut für Europäische Politik, Oktober 2006 (unveröffentlicht), S. 43.

34 Vgl. Müller-Brandeck-Bocquet: Frankreichs Europapolitik, S. 267.

35 Contrat de législature 2007-2012, abrufbar unter: http://viphttp.yacast.net/ump/projet-ump2007.pdf (letzter Zugriff: 28.12.2006).

36 Neue Zürcher Zeitung: Klares Bekenntnis Sarkozys zur EU, 09.09.2006. 
wo es um eine Art Übertragung eigener nationaler französischer Konzepte besonders im Bereich der Sozialpolitik auf die europäische Ebene geht.

Im Wahlkampf lässt Ségolène Royal außerdem seit ihrer Kür zur Kandidatin bisweilen souveränistische Töne verlauten. In ihrer Antrittsrede zur Kandidatin der PS betonte sie die Bedeutung der ,Nation“ und Frankreichs als Zentrum des politischen Handels. In ihrer Rede forderte sie, dass die Linke den Begriff ,Nation“ wieder positiv für sich definieren müsse und ihn nicht der Rechten überlassen dürfe. ${ }^{37}$ Jean-Pierre Chevènement, der 1993 aus der PS austrat um das Mouvement des Citoyens in eine links-nationale Partei umzubilden, war 2002 als Präsidentschaftskandidat angetreten und hatte 5,3 Prozent der Stimmen erreicht. Als vehementer Gegner des Verfassungsvertrags verzichtete er im Dezember zugunsten von Royal auf eine eigene Kandidatur und bekam im Gegenzug sichere Mandate für Kandidaten seiner Partei bei den Parlamentswahlen zugesprochen. Sein Einfluss auf den inhaltlichen Verlauf des Wahlkampfes bleibt abzuwarten.

In Kontinuität mit traditionellen Ideen der französischen Europapolitik wird die europäische Einigung auf der einen Seite zwar befürwortet, auf der anderen Seite wird sie jedoch als Möglichkeit betrachtet, eben gerade die Besonderheit Frankreichs zu erhalten. ${ }^{38}$ Ein Verfassungsvertrag im Sinne eines kleinsten gemeinsamen gesamteuropäischen Nenners scheint nicht dem französischen Politikverständnis zu entsprechen.

\section{Sozial- und Wirtschaftspolitik - dominierendes europapolitisches Thema}

Das dominante europapolitische Thema des Wahlkampfes ist die Wirtschafts- und Sozialpolitik. Die sozialen Krisenbewegungen, wie die Probleme in den Vorstädten im November 2005 oder die Massenbewegung gegen den so genannten Contrat de Première Embauche (CPE), einen speziellen Vertrag, der den Kündigungsschutz für Berufsanfänger aufgehoben hätte, sowie eine steigende Anzahl von Protestwählern zeigen, dass in der französischen Bevölkerung Ängste in Bezug auf steigenden Wettbewerb und Sozialdumping existieren. Bereits in der Debatte um den Verfassungsvertrag hatten sozio-ökonomische Fragestellungen einen hohen Stellenwert. So gaben 31 Prozent der Befragten aus dem Lager der ,Nein“Wähler an, ihre Ablehnung resultiere aus der Einschätzung, dass der Verfassungsvertrag negative Konsequenzen für die Arbeitsplatzsituation in Frankreich habe. Weitere 19 Prozent gaben an, Europa sei zu ,liberal' während 16 Prozent befanden, dass es nicht genug sozialpolitische Elemente im Verfassungsvertrag gebe. ${ }^{39}$

Die europapolitische Debatte zu sozial- und wirtschaftspolitischen Themen wird in beiden Lagern von Schlagwörtern beherrscht wie ,Sozialdumping' innerhalb der (erweiterten) Europäischen Union, der Forderung nach einer ,préférence communautaire“ als aktiver Politik zum Schutz der europäischen Wirtschaft nach außen, und der Forderung nach einem „gouvernement économique“ mit der wirtschaftspolitischen Zielsetzung von Beschäftigung und Wachstum in der Eurogruppe ${ }^{40}$, wenn sich die Vorschläge im Detail auch unterschied-

37 Discours de Ségolène Royal au Congrès d'investiture du Parti Socialiste à la Mutualité, 26.11.2006, abrufbar unter: http://www.desirsdavenir.org/index.php?c=interventions\&id=461 (letzter Zugriff: 28.12.2006).

38 Brincker: Country study: Leitbilder for the enlarged European Union in France, S. 15.

39 Europäische Kommission: La Constitution européenne: sondage post-référendum en France, Eurobarometer 171, 2005, S. 17.

40 Vgl. Parti Socialiste: Réussir ensemble le changement, S. 31; LeMonde.fr: Texte élaboré par la commission chargée du programme du parti gaulliste pour les éléctions législatives de 2007, 14.11.2006, abrufbar unter: http:// www.lemonde.fr/web/article/0,1-0@2-823448,36-834320@51-834373,0.html (letzter Zugriff: 16.11.2006). 
lich gestalten. Dies knüpft an die französischen Vorstellungen einer stärkeren politischen Kontrolle der Europäischen Zentralbank (EZB) an.

In Anbetracht der öffentlichen Debatte nach dem Verfassungsreferendum schwenkt sogar der vergleichsweise ,liberale ' Politiker Nicolas Sarkozy auf einen protektionistischen Diskurs um. ${ }^{41}$ Die EU solle die Aufgabe des ,Schutzes“442 der Bürger vor den negativen Folgen der Globalisierung übernehmen. ${ }^{43}$ Sarkozy hatte sich ursprünglich für größere Wettbewerbsfähigkeit, ein Wahrnehmen der Globalisierung als Chance und das angelsächsische EU-Leitbild ausgesprochen. ${ }^{44}$

Im Zentrum des europapolitischen Teils des Wahlprogramms der PS steht ein „Plan zur Wiederbelebung Europas“, dessen wichtigster Vorschlag ein „Sozialvertrag“ ist, der eine Harmonisierung der Sozialpolitiken der Mitgliedstaaten, eine Richtlinie zum Öffentlichen Dienst und die Verabschiedung von ,sozialpolitischen Konvergenzkriterien“ zwischen den Mitgliedstaaten zum Inhalt hätte. ${ }^{45}$ Aus Sicht der PS gefährdet der liberale Kurs der Europäischen Union das Projekt einer ,solidarischen Integration“. ${ }^{46}$ Die Frage, inwieweit man eine liberale Wirtschaftspolitik der EU mitträgt, ist innerhalb der PS in der Vergangenheit schon häufig diskutiert worden. Die oben erwähnte Verschiebung der dominierenden europapolitischen Konfliktlinie basierte unter anderem auf einer Spaltung innerhalb der PS zwischen Anhängern der sozialistischen Tradition und der Sozialdemokratie. ${ }^{47}$ Nicht alle Mitglieder und Wähler der sozialistischen Partei teilen die sozialdemokratischen Prinzipien Europas. Gemäß der marxistischen und trotzkistischen Tradition der Linken in Frankreich haben sich Teile der Wählerschaft der PS noch nicht von ihren sozialistischen Ideen entfernt und halten selbst eine regulierte Marktwirtschaft nicht für das beste ökonomische System. ${ }^{48}$ Bereits unter Mitterrand hatte sich bezüglich der künftigen französischen Europapolitik zwischen diesen beiden Strömungen ein Konflikt innerhalb der Partei entzündet.

Die PS war daher bis zum Sommer 2006 vor allem darum bemüht, wieder eine einheitliche Position zu finden. Die Spaltung scheint nun teilweise überwunden, wichtige Vertreter des ,Nein ' unterstützen aktuell Ségolène Royal, so beispielsweise Arnaud Montebourg und Vincent Peillon, die Köpfe der liberalismuskritischen und linksgerichteten Strömung der Parti Socialiste namens Nouveau Parti Socialiste. Sie kann jedoch jederzeit wieder aufbrechen, da eine endgültige Entscheidung über die Ausrichtung der Partei in Richtung Sozialdemokratie immer noch bevorsteht.

\section{Vorstellungen zur europäischen Außen- und Sicherheitspolitik - ,l'Europe puissance oder transatlantischer Frühling?}

In Bezug auf die Außen- und Sicherheitspolitik ist erstaunlich, mit welcher Einhelligkeit sowohl die Sozialisten wie auch die Konservativen eine stärkere gemeinsame Außen- und Sicherheitspolitik als eines der Zukunftsprojekte der Europäischen Union betonen. Ein sol-

41 Vgl. Institut für Europäische Politik (Hrsg.): EU-25 Watch No. 2, Berlin 2006, S. 69.

42 Vgl. Rede des Parteivorsitzenden Nicolas Sarkozy „La France dans la mondialisation“, Saint-Etienne, 09.11.2006, abrufbar unter: http://www.u-m-p.org/site/GrandDiscoursAffiche.php?IdGrandDiscours=269 (letzter Zugriff: 10.11. 2006).

43 Vgl. Institut für Europäische Politik (Hrsg.): EU-25 Watch No. 2, S. 69.

44 Vgl. Brincker: Country study: Leitbilder for the enlarged European Union in France, S. 61.

45 Vgl. Parti Socialiste: Réussir ensemble le changement, S. 31-32.

46 Vgl. Parti Socialiste: Réussir ensemble le changement, S. 31.

47 Vgl. Brantner/Knoll: Constitution, ,attac-ed“.

48 Florence Deloche-Gaudez/Christian Lequesne: Frankreich, in: Wolfgang Wessels/Werner Weidenfeld (Hrsg.): Jahrbuch der Europäischen Integration 2005, Baden-Baden 2006, S. 329-336, hier S. 331. 
ches Projekt reflektiert das seit de Gaulle existierende Leitbild der ,Europe puissance ${ }^{49}$, das in der Europäischen Union einen Akteur auf der internationalen Bühne sieht.

Dabei sei jedoch auf einen Unterschied hingewiesen, den Sylvie Goulard schon im Wahlkampf 2000 zwischen der damaligen Rassemblement pour la République (RPR) und der PS ausmachte. Die RPR spreche sich für eine stärkere Außenpolitik der Europäischen Union aus, um diese in eine intergouvernementale Richtung zu stärken. ${ }^{50}$ Auch in der jetzigen Debatte ist nicht eindeutig, inwieweit die UMP und Sarkozy wirklich Ansätze einer Vergemeinschaftung in diesem Feld befürworten.

Das Parteimanifest der PS fordert eine verstärkte Zusammenarbeit im Bereich der Verteidigungspolitik, ausgehend von den drei großen Mitgliedstaaten: Frankreich, Großbritannien und Deutschland. Es bleibt vage in Bezug auf deren Umsetzung und der Frage, ob dies innerhalb oder außerhalb der bestehenden Verträge geschehen solle.

Die Unterschiede zwischen Ségolène Royal und Nicolas Sarkozy liegen vorwiegend in der Einschätzung der Rolle der Europäischen Union gegenüber den USA. Eines der wichtigsten Leitbilder in der französischen Europapolitik ist die Vision eines starken Europas gegenüber den USA $^{51}$ und Frankreichs Rolle als wichtiger außenpolitischer Akteur als Nuklearmacht und mit einem Sitz im Sicherheitsrat der Vereinten Nationen. ${ }^{52}$ In dieser Tradition steht auch weiterhin Royal. Sie vertritt das ,europäische (also autonome) Europa“"53 als Alternative zum transatlantischen Europa.

Im Gegensatz dazu sieht Nicolas Sarkozy in den USA einen Partner. Auch wenn er keine „Hörigkeit“" gegenüber den USA will, so sieht er doch zum Beispiel retrospektiv die Androhung des Vetos durch Frankreich in der Irakfrage als überzogen an. ${ }^{54}$ In einem Interview mit der Tageszeitung Le Monde führte er aus, dass die USA auch ein Modell für Frankreich sein könne. Eine Reise in die USA Anfang September wurde auch prompt als pro-amerikanisches Signal verstanden. Sarkozy nimmt damit eine Position ein, die in Frankreich nicht gerade populär ist und nicht der französischen Tradition entspricht.

\section{Erweiterung und Grenzen der Europäischen Union - das Querschnittsthema}

Die Erweiterung der Europäischen Union ist in der französischen europapolitischen Debatte ein Querschnittsthema. Mit der Erweiterung verbindet sich in Frankreich die Sorge, ökonomisch, machtpolitisch und geografisch marginalisiert zu werden. ${ }^{55}$

Seit einer Verfassungsänderung unterliegt die Ratifikation von Beitritten zur Europäischen Union in Frankreich einem Referendum. ${ }^{56}$ Nicolas Sarkzoy hat sich in seiner Europarede am 8. September 2006 eindeutig gegen eine Beitrittsperspektive für die Türkei ausgesprochen. Er sprach sich gegen ein „Europa ohne Grenzen“ aus und forderte eine Debatte um einen ,geografischen und politischen Rahmen“ der Europäischen Union. Die Voraussetzung für weitere Erweiterungen sei die Aufnahmefähigkeit der Union, die er als nicht ,unendlich ausdehnbar“ qualifiziert. Für Sarkozy können der Europäischen Union solche Staaten beitreten, die „klar dem europäischen Kontinent angehören“. Für andere Staaten,

49 Vgl. Sabine Jung: Europa, made in France: eine Analyse des politischen Diskurses Frankreichs zur Zukunft der Europäischen Gemeinschaft: von den Anfängen bis heute, Baden-Baden 1999.

50 Vgl. Goulard: Französische Europapolitik und öffentliche Debatte, S. 6.

51 Vgl. Stark: Frankreichs Rolle in der Welt.

52 Brincker: Country study: Leitbilder for the enlarged European Union in France, S. 13.

53 Vgl. Stark: Frankreichs Rolle in der Welt.

54 Le Monde : J'aime l'énergie et la fluidité de l'Amérique, Interview mit Nicolas Sarkozy, 09.09.2006.

55 Brincker: Country study: Leitbilder for the enlarged European Union in France, S. 12.

56 Vgl. La Constitution du 4 octobre 1958, Art. 88-5. 
darunter die Türkei, die „Nachbarn sind, ohne Europäer zu sein“, fordert Sarkozy die Abkehr von der Vision einer Vollmitgliedschaft. Stattdessen sollen diese Staaten in einer privilegierten Partnerschaft an die EU gebunden werden. ${ }^{57}$ Dies ist auch die von der UMP in ihrem Wahlkampf vertretene Position. Die Kandidaten der extremen Rechten, wie der nationalkonservative Villiers und der rechtsextreme Le Pen, sprechen sich gegen den Beitritt der Türkei aus.

Auch die sozialistische Partei steht weiteren Erweiterungen skeptisch gegenüber. Sie fordert in ihrem Programm eine „Klärung und Definition der Grenzen Europas“. Getroffene Beschlüsse sind zwar einzuhalten, die Erweiterung solle sich jedoch auf die Prüfung der bisherigen offiziellen Kandidaturen beschränken. Über die Grenzen Europas hinaus sollen „strategische Partnerschaften“ angestrebt werden. ${ }^{58}$

\section{Der deutsch-französische Motor - Relikt einer anderen Generation?}

Einer der Hauptpfeiler französischer Europapolitik seit de Gaulle war die Einbindung Deutschlands in die europäischen Strukturen. ${ }^{59}$ Nachdem das deutsch-französische Tandem unter Chirac und Schröder, besonders unter dem Eindruck des Irakkonfliktes, einen erneuten Auftrieb erlebte, scheint es seit dem Amtsantritt von Kanzlerin Merkel geschwächt, da sie einen transatlantischeren Kurs eingeschlagen hat und von Anfang an ihren Willen bekundete, auch andere Partner stärker miteinzubeziehen.

Weder in den Debattenbeiträgen von Sarkozy noch von Royal taucht die besondere Bedeutung des deutsch-französischen Motors auf. Sarkozy zieht sogar dessen Leistungsfähigkeit in Zweifel. Der Motor sei alt und überholt. Stattdessen schlug er 2004 eine Hinwendung zu Großbritannien und Spanien beziehungsweise auch Italien und Polen vor. ${ }^{60}$ Als neuen Motor bezeichnet er stattdessen Kooperationen von Staaten, die bereit sind, je nach Politikfeld im Sinne einer variablen Geometrie gemeinsam voranzuschreiten. ${ }^{61}$

Im Gegensatz zur einem altgedienten Politiker wie beispielsweise Dominique StraussKahn, bei dem die Wiederbelebung des deutsch-französischen Motors im Zentrum einer gelungenen Europapolitik stand und in dessen ,klinischem Tod“ er einen der Hauptgründe für die aktuelle Krise der EU sah ${ }^{62}$, könnte sich eine neue - wenn auch gar nicht unbedingt jüngere - Politikergeneration Royal/Sarkozy von einem privilegierten deutsch-französischen Verhältnis abwenden und neue Verbündete suchen.

In Bezug auf den deutsch-französischen Motor wird so in nächster Zeit mehr denn je fraglich bleiben, welchen Stellenwert und welche Zugkraft dieser in einer EU von 27 Staaten hat und haben wird. Es wird mühsam sein, tragbare Koalitionen mit anderen Mitgliedstaaten zu schmieden. Deutschland und Frankreich konnten in einer EU mit 15 Mitgliedstaaten ihre Kompromissfähigkeit gerade aus der Tatsache generieren, dass ihre Positionen häufig relativ weit auseinander lagen. In Anbetracht einer steigenden Heterogenität der erweiterten Union rücken deutsch-französische Positionen bisweilen enger zueinander und sind für die anderen Mitgliedstaaten unter Umständen dann weniger anschlussfähig. Gegensatzpaare bil-

57 Vgl. Rede von Nicolas Sarkozy vor den Amis de l'Europe.

58 Parti Socialiste: Réussir ensemble le changement.

59 Vgl. Wichard Woyke: Deutsch-französische Beziehungen seit der Wiedervereinigung. Das Tandem fasst wieder Tritt, Opladen 2000, S. 15.

60 Müller-Brandeck-Bocquet : Frankreichs Europapolitik, S. 271.

61 Vgl. Rede von Nicolas Sarkozy vor den Amis de l'Europe, S. 5.

62 Vgl. Vorschläge von Dominique Strauss-Kahn zu Europa, abrufbar unter: http://www.dsk2007.net/-Mes-propositions-.html (letzter Zugriff: 20.11.2006). 
den sich neu. ${ }^{63}$ Zusammen gesehen wirft dies die Frage auf, ob das deutsch-französische Paar als Relikt einer alten Politikergeneration gelten kann.

\section{Zwischen Wandel und Kontinuität}

In Bezug auf die Ziele und Leitbilder französischer Europapolitik zeichnet sich ein Wandel in Richtung auf eine neue Akzeptanz der Europäischen Union als politischer Handlungsrahmen und für supranationale Elemente des institutionellen Gefüges der EU in der öffentlichen Debatte ab. Auch der nächste Präsident wird sich für ein starkes Frankreich in einem zu stärkenden Europa einsetzen - für letzteres jedoch nur, so lange ein starkes Europa nicht französische Traditionen der Sozial-, Wirtschafts- und Außenpolitik schwächt.

Obwohl beide Kandidaten für sich einfordern, ,neuartig' zu sein und einer , neuen Generation ' von Politikern anzugehören, sind jedoch Kontinuitäten in Bezug auf europapolitische Leitbilder unverkennbar. Zwar sah es zeitweilig so aus, als könne mit Sarkozy ein liberalerer Diskurs in die französische Wirtschaftspolitik einziehen, doch ist nach dem Referendumsdebakel auch die UMP zu einem Diskurs zurückgekehrt, der mehr, Schutz durch die Europäische Union einfordert. Bei Royal scheint es, als müsse sie sich nach der schwierigen Zerreißprobe ihrer Partei erst recht auf bekannte Zielorientierungen zurückbesinnen. Im Bereich der Außenpolitik wird sich noch zeigen müssen, ob es mit einem Präsidenten Sarkozy eine neue transatlantische Annäherung geben könnte, was jedoch aufgrund der Stimmungslage in der Öffentlichkeit zweifelhaft scheint.

Beide Kandidaten führen einen Wahlkampf, der häufig als ,populistisch ' beschrieben wird. Sie versuchen, auch die Stimmen der Extreme zu gewinnen, die immerhin meist jeweils 15 bis 20 Prozent der Stimmen erhalten. Gleichzeitig beanspruchen beide Kandidaten jedoch auch für sich, für einen , pragmatischen ' Politikstil zu stehen. Es bleibt demnach abzuwarten, was von der Wahlkampfrhetorik an konkreter Politik übrig bleibt.

Die fehlende diskursive Unterstützung der Idee einer auf Kompromissen fußenden Europäischen Union birgt die Gefahr, dass weiterhin bei den französischen Bürgern unrealisierbare Hoffnungen genährt werden, die nur enttäuscht werden können. Dies könnte zu einer Negativ-Spirale führen, die langfristig die Akzeptanz der EU in Frankreich unterminieren könnte.

Der Beitrag ist in Zusammenhang mit dem Forschungsprojekt „Europapolitische Leitbilder in der erweiterten Europäischen Union “ entstanden, das vom Institut für Europäische Politik, dem Jean Monnet Lehrstuhl für Politische Wissenschaft und Europäische Fragen der Universität zu Köln sowie der Karls Universität Prag durchgeführt und von der VolkswagenStiftung gefördert wird.

63 Vgl. Daniela Schwarzer : Deutschland und Frankreich - Duo ohne Führungswillen. Das bilaterale Verhältnis in der europäischen Union, SWP-Studie S15/2006, S. 25-26. 